\title{
Enhancing the lateral-flow immunoassay for viral detection using an aqueous two-phase micellar system
}

\author{
Foad Mashayekhi $\cdot$ Ricky Y. T. Chiu • \\ Alexander M. Le • Felix C. Chao • Benjamin M. Wu • \\ Daniel T. Kamei
}

Received: 23 July 2010 /Revised: 7 September 2010 / Accepted: 8 September 2010 /Published online: 24 September 2010

(C) The Author(s) 2010 This article is published with open access at Springerlink.com

\begin{abstract}
Availability of a rapid, accurate, and reliable point-of-care (POC) device for detection of infectious agents and pandemic pathogens, such as swine-origin influenza A (H1N1) virus, is crucial for effective patient management and outbreak prevention. Due to its ease of use, rapid processing, and minimal power and laboratory equipment requirements, the lateral-flow (immuno)assay (LFA) has gained much attention in recent years as a possible solution. However, since the sensitivity of LFA has been shown to be inferior to that of the gold standards of pathogen detection, namely cell culture and real-time PCR, LFA remains an ineffective POC assay for preventing pandemic outbreaks. A practical solution for increasing the sensitivity of LFA is to concentrate the target agent in a solution prior to the detection step. In this study, an aqueous two-phase micellar system comprised of the nonionic surfactant Triton X-114 was investigated for concentrating a model virus, namely bacteriophage M13 (M13), prior to LFA. The volume ratio of the two coexisting micellar phases was manipulated to concentrate M13 in the top, micelle-poor phase. The concentration step effectively improved the M13 detection limit of the assay by tenfold from $5 \times 10^{8}$ plaque forming units $(\mathrm{pfu}) / \mathrm{mL}$ to $5 \times 10^{7} \mathrm{pfu} /$ $\mathrm{mL}$. In the future, the volume ratio can be further manipulated to yield a greater concentration of a target virus and further decrease the detection limits of the LFA.
\end{abstract}

Electronic supplementary material The online version of this article (doi:10.1007/s00216-010-4213-7) contains supplementary material, which is available to authorized users.

F. Mashayekhi • R. Y. T. Chiu • A. M. Le · F. C. Chao •

B. M. Wu D. T. Kamei $(\square)$

Department of Bioengineering, University of California,

5121 Engineering V, 420 Westwood Plaza,

Los Angeles, CA 90095-1600, USA

e-mail:kamei@seas.ucla.edu
Keywords Lateral-flow immunoassay Aqueous two-phase micellar systems $\cdot$ Point-of-care $\cdot$ Viral concentration and detection $\cdot$ Diagnostics

\section{Introduction}

The recent outbreak of swine-origin influenza A (H1N1) virus infection [1] highlighted the need for a means to rapidly and accurately detect infectious agents and pandemic pathogens at the point-of-care (POC), which in turn could result in better patient management, such as timely use of appropriate antiviral treatments and isolation of confirmed cases, to aid in preventing outbreaks $[2,3]$. One detection method that has gained much attention in recent years due to its ease of use, rapid time to result, and minimal power and laboratory equipment requirements is the lateral-flow (immuno)assay (LFA). LFA utilizes a test strip that collects a sample through lateral flow, and detects the presence of a target molecule through its specific antibody labeled with a colorimetric indicator. While LFA has been used to detect a wide range of biomolecules [4], its sensitivity in detecting viruses has been shown to range from $19 \%$ to $96 \%$ compared to the gold standards, namely viral culture and real-time PCR [3, 5-9]. Although LFA is superior to viral culture and real-time PCR in terms of ease of use and rapid time to result, its typically low sensitivity in detecting pathogens and viruses limits its effectiveness as a reliable POC assay for preventing pandemic outbreaks. Therefore, the sensitivity of LFA for detection of infectious viruses must first be improved before it can reliably be used as an effective POC solution.

One approach to achieve a higher sensitivity for LFA is to improve the assay itself. Another approach is to concentrate the target biomolecule prior to the detection step. The focus of this work is on the latter approach, and 
we investigated concentrating a target virus using an aqueous two-phase system (ATPS) prior to its detection via LFA. Although ATPS have also been previously studied, the work reported here is novel and significant, since it represents the first time the established technologies of LFA and ATPS have been combined. In fact, ATPS, which can be formed with micelles (aqueous two-phase micellar system, ATPMS), polymers, or a combination of the two, have generally been examined for large-scale biotechnological applications [10-16] and not small-scale diagnostic applications. ATPS are very appropriate for a POC device, since they are easy to use, can be rapid, and are scalable (to require minimal sample volume). Furthermore, ATPS also do not require any laboratory equipment and are low in cost compared to chromatography [17-19].

In this proof-of-principle study, an ATPMS comprised of the nonionic surfactant Triton X-114 and phosphate-buffered saline (PBS) was investigated for concentrating a model virus, namely bacteriophage M13 (M13). In an aqueous solution at concentrations above their critical micelle concentration, the surfactant molecules form micelles [20]. The Triton X-114 micellar system exhibits a homogeneous, isotropic phase at low temperatures. Upon increasing the temperature, the solution undergoes a macroscopic phase separation to yield a top, micelle-poor phase and a bottom, micelle-rich phase. Biomolecules would then distribute, or partition, unevenly between the two phases based on their physico-chemical characteristics, such as hydrophobicity [21] and size [22]. First, we wanted to gain an understanding of the main driving forces for M13 partitioning in the Triton X-114 micellar system to assess the predictability of the M13 partitioning behavior. Accordingly, the experimentally measured partitioning behavior of M13 was compared with our theoretical predictions based on a model developed recently for the partitioning of cylindrical biomolecules in ATPMS [16]. Next, M13 was concentrated in the top, micelle-poor phase by manipulating the volume ratio (the volume of the top, micelle-poor phase divided by that of the bottom, micelle-rich phase). After ensuring that we could concentrate M13 in the ATPMS in a predictive manner, we developed an LFA for the detection of M13 in-solution. Once the detection limit of the immunoassay was established, M13 was concentrated by utilizing the ATPMS prior to the detection step to investigate the effect of the concentration step on the immunoassay's detection limit.

\section{Materials and methods}

Culturing and quantifying M13

Escherichia coli bacteria (American Type Culture Collection, ATCC, Manassas, VA) were incubated in $6 \mathrm{~mL}$ of lysogeny broth (10 g/L tryptone (BD, Franklin Lakes, NJ), $5 \mathrm{~g} / \mathrm{L}$ yeast extract $(\mathrm{BD})$, and $10 \mathrm{~g} / \mathrm{L} \mathrm{NaCl}$ ) at $37{ }^{\circ} \mathrm{C}$ and $240 \mathrm{rpm}$ in a shaker incubator for $12 \mathrm{~h}$. In order to culture M13 (ATCC), $10 \mu \mathrm{L}$ of the stock M13 solution was added to the cultured bacteria solution. The bacteria solution was then incubated in a shaker incubator at $37{ }^{\circ} \mathrm{C}$ and $240 \mathrm{rpm}$ for $5 \mathrm{~h}$. The solution was then centrifuged at $4{ }^{\circ} \mathrm{C}$ and $8,000 \times g$ for $15 \mathrm{~min}$ to remove the bacteria. The supernatant containing M13 was collected and filtered using a $0.22 \mu \mathrm{m}$ syringe filter (Millipore, Billerica, MA).

As described previously [23], the plaque assay was used to quantify the concentration of M13 in-solution, and therefore, the concentration of M13 is reported as plaque forming units (pfu) per $\mathrm{mL}$. All reagents and materials were purchased from Sigma-Aldrich (St. Louis, MO) unless otherwise noted.

\section{Partitioning and concentrating M13}

For each M13 partitioning experiment, three identical $3.5 \mathrm{~mL}$ Triton X-114 solutions in Dulbecco's PBS (Invitrogen, $\mathrm{pH} 7.4$, containing $1.47 \mathrm{mM} \mathrm{KH} \mathrm{PO}_{4}, 8.10 \mathrm{mM}$ $\mathrm{Na}_{2} \mathrm{HPO}_{4}, 137.93 \mathrm{mM} \mathrm{NaCl}, 2.67 \mathrm{mM} \mathrm{KCl}$, and $0.49 \mathrm{mM}$ $\mathrm{MgCl}_{2}$ ) were prepared. M13 was added to the solutions at a concentration of $10^{8} \mathrm{pfu} / \mathrm{mL}$. In order to ensure that each solution is in one phase prior to phase separation, the solutions were equilibrated at $4{ }^{\circ} \mathrm{C}$ prior to the addition of M13. Once M13 was added, the solutions were mixed and placed in a water bath set at the appropriate temperature which yielded a volume ratio equal to approximately 1 . The operating conditions (i.e., temperature and initial surfactant concentration) are listed in Table S1 in the Electronic Supplementary Material. After incubating the three solutions in the water bath for $18 \mathrm{~h}$, the two coexisting micellar phases were withdrawn carefully using syringe and needle sets, and the concentration of M13 in each of the two phases was determined using the plaque assay. Each partitioning experiment, which involved triplicate solutions, was repeated at least twice.

By altering the volume ratio of the partitioning experiments, M13 can be concentrated in the top, micelle-poor phase. The same protocol described above for partitioning M13 was used, except that the initial Triton X-114 surfactant concentrations and operating temperatures were varied in order to achieve the desired volume ratios. The operating conditions and resulting volume ratios are listed in Table S2 in the Electronic Supplementary Material.

Preparing colloidal gold probes

The colloidal gold nanoparticles were prepared according to Frens [24]. The colloidal gold-antibody probe was prepared as described by Horisberger and Clerc [25]. Briefly, the $\mathrm{pH}$ 
of a 2.5-mL colloidal gold nanoparticle solution was adjusted to $\mathrm{pH} 9$ using $0.1 \mathrm{M} \mathrm{NaOH}$. Subsequently, $40 \mu \mathrm{g}$ of anti-M13 mouse monoclonal antibody (Abcam Inc., Cambridge, MA) at a concentration of $0.2 \mathrm{mg} / \mathrm{mL}$ was added to the colloidal gold solution and mixed for $10 \mathrm{~min}$ on a shaker. To prevent nonspecific binding of other proteins to the surfaces of the colloidal gold nanoparticles, $250 \mu \mathrm{L}$ of $10 \% \mathrm{w} / \nu$ bovine serum albumin (BSA) was added to the mixture and mixed for $15 \mathrm{~min}$ on a shaker to block all excess surfaces on the colloidal gold nanoparticles. The mixture was then centrifuged for $30 \mathrm{~min}$ at $4{ }^{\circ} \mathrm{C}$ and $9,000 \times g$ to remove free antibody and BSA. The pellet, which contained the colloidal gold nanoparticles, was resuspended in $375 \mu \mathrm{L}$ of $0.1 \mathrm{M}$ sodium borate buffer, $\mathrm{pH} 9$.

\section{Preparing LFA test strips}

There are two different approaches for the LFA: the sandwich assay and the competition assay. In this study, we implemented the sandwich assay. As shown schematically in Fig. 1, in the sandwich assay, antibodies specific for the target of interest are immobilized on a nitrocellulose membrane in the form of a line, called the test line. Secondary antibodies against the primary antibody are also immobilized on the nitrocellulose membrane in the form of a line, called the control line. In LFA, a sample first comes into contact with the colloidal gold probes. If the target molecules are present in the sample, they would first bind to their specific antibodies immobilized on the colloidal gold nanoparticles. Subsequently, as the colloidal gold nanoparticles move up the LFA strip, the target molecules present in the colloidal gold-antibody-target molecule complexes would bind to their specific antibodies immobilized on the test line. Due to the presence of "trapped" colloidal gold particles, which exhibit a purplish red color, a visual band is formed at the test line that indicates a positive result (Fig. 1a). Alternatively, if the target molecule

\section{(a)}
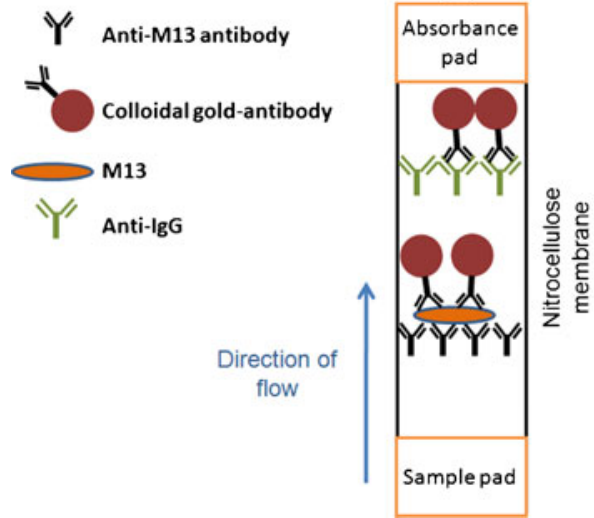

(b)

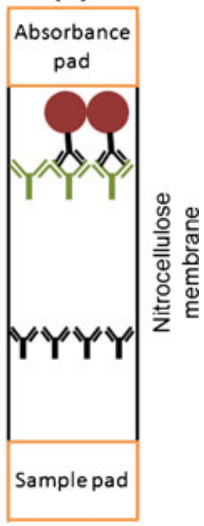

Fig. 1 Schematic representations of a positive and $\mathbf{b}$ negative results for the LFA using the sandwich mechanism is not present in the solution, the colloidal gold-complexed antibodies would not be "trapped" by the immobilized antibodies at the test line. This indicates a negative result (Fig. 1b). Furthermore, regardless of the presence of the target molecule in the sample, colloidal gold-complexed antibodies would bind and get "trapped" by the immobilized secondary antibodies on the control line, which indicates a valid test. The LFA test strips used in this study were prepared using a similar approach to that of Schuurs and coworkers [26].

\section{Performing LFA on M13}

The operating condition that was used for performing the concentration step prior to the detection step resulted in M13 being concentrated in the top, micelle-poor phase with a Triton X-114 concentration of $0.065 \% w / w$ in PBS. Therefore, to be consistent between the immunoassays performed with or without the concentration step, solutions of M13 in $0.065 \% w / w$ Triton X-114 in PBS were used to perform the LFA without the concentration step. The LFA was performed as follows: Solutions containing varying M13 concentrations diluted in $0.065 \% w / w$ Triton X-114 in PBS were first prepared. Forty-five microliters of the M13 solutions were then added to $10 \mu \mathrm{L}$ of the colloidal gold probes solution and $25 \mu \mathrm{L}$ of test buffer $(0.2 \%$ BSA, $0.3 \%$ Tween $20,0.2 \%$ sodium azide, $0.1 \%$ polyethylene glycol, 0.1 M Trizma base, $\mathrm{pH} 8$ ), which was used to facilitate the flow of the samples through the test strips. The resulting solutions were mixed, and incubated for $5 \mathrm{~min}$ before a test strip was dipped vertically into each solution so that only the sample pad would come in contact with the solution. After $20 \mathrm{~min}$, the test strips were taken out of the solution, and an image of each strip was immediately taken by a Canon EOS 1000D camera (Canon U.S.A., Inc., Lake Success, NY).

\section{Combining ATPMS with LFA}

To combine the concentration step with the detection step, M13 was first concentrated following the same protocol as mentioned previously in Partitioning and Concentrating M13. Solutions containing 9.50\% $w / w$ Triton X-114 in PBS were used, and the solutions were incubated at $26.0{ }^{\circ} \mathrm{C}$ for $18 \mathrm{~h}$. At these operating conditions, a volume ratio of $1 / 9$ (0.111) was obtained. In addition, various amounts of M13 were added to each solution to obtain appropriate initial concentrations of M13. After phase separation, the top phases were withdrawn carefully using syringe and needle sets. The LFA was performed as described in Performing LFA on M13, except instead of using $45 \mu \mathrm{L}$ of the M13 solutions diluted in $0.065 \% w / w$ Triton X-114 in PBS, $45 \mu \mathrm{L}$ of the withdrawn top, micelle-poor phases were used. 


\section{Results and discussion}

Partitioning M13 in ATPMS

The partitioning behavior of a biomolecule in an ATPMS is quantified by evaluating the partition coefficient, $K_{\mathrm{bm}}$, which is defined as follows:

$K_{\mathrm{bm}} \equiv \frac{C_{\mathrm{bm}, \mathrm{t}}}{C_{\mathrm{bm}, \mathrm{b}}}$ where $C_{\mathrm{bm}, \mathrm{t}}$ and $C_{\mathrm{bm}, \mathrm{b}}$ are the concentrations of the biomolecule in the top and bottom phases, respectively. Based on its shape, M13 can be modeled as a cylindrical biomolecule. We had previously extended a model, which was developed by Blankschtein and coworkers [22, 27] for the partitioning of spherical, water-soluble proteins in ATPMS, to cylindrical biomolecules (cb). This model incorporates the repulsive, steric, excluded-volume interactions that operate between the cylindrical biomolecule and the cylindrical micelles, and the following expression was derived for the partition coefficient of a cylindrical biomolecule, such as M13 [16]:

$K_{\mathrm{cb}}^{\mathrm{EV}}=\exp \left[-\left(\phi_{\mathrm{t}}-\phi_{\mathrm{b}}\right)\left(1+\frac{l_{\mathrm{cb}} R_{\mathrm{cb}}^{2}}{l_{\mathrm{m}} R_{\mathrm{m}}^{2}}+\frac{1}{2}\left[\frac{R_{\mathrm{m}}\left(l_{\mathrm{m}}+R_{\mathrm{m}}\right)\left(l_{\mathrm{cb}}+\pi R_{\mathrm{cb}}\right)+R_{\mathrm{cb}}\left(l_{\mathrm{cb}}+R_{\mathrm{cb}}\right)\left(l_{\mathrm{m}}+\pi R_{\mathrm{m}}\right)}{l_{\mathrm{m}} R_{\mathrm{m}}^{2}}\right]\right)\right]$

where $\Phi_{\mathrm{t}}$ and $\Phi_{\mathrm{b}}$ are the surfactant volume fractions in the top and bottom phases, respectively, $R_{\mathrm{cb}}$ and $R_{\mathrm{m}}$ are the cross-sectional radii of the biomolecule and micelles, respectively, and $l_{\mathrm{cb}}$ and $l_{\mathrm{m}}$ are the lengths of the biomolecule and micelles, respectively. The crosssectional radius of M13 was estimated to be $7 \mathrm{~nm}$, and its length was estimated to be $900 \mathrm{~nm}$ [28]. The cross-sectional radius of Triton X-114 micelles was estimated to be $23.4 \AA$ [16], while their lengths were estimated to be between $10 \mathrm{~nm}$ and $1 \mu \mathrm{m}$ [29-33]. Based on Eq. 2, the values for the dimensions of M13 and Triton X-114 micelles, and the one-to-one correspondence between operating temperature and $\left(\Phi_{\mathrm{t}}-\Phi_{\mathrm{b}}\right)$ found previously [16], extremely large $(\gg>1,000)$ M13 partition coefficients as a function of temperature were predicted. However, as shown previously for the partitioning behavior of DNA fragments and spherical viruses in ATPMS [16, 34], the entrainment of micelle-poor domains in the macroscopic micelle-rich phase has a significant impact on the partitioning behavior of such large hydrophilic macromolecules.

Due to the small density difference and interfacial tension between the micelle-rich and micelle-poor domains, macroscopic phase separation equilibrium is not attained even after waiting a long time. As a result, some micellepoor domains are entrained in the macroscopic micelle-rich phase, and similarly, some micelle-rich domains are entrained in the macroscopic micelle-poor phase. If M13 partitions extremely into the micelle-poor domains, as predicted by the model, the concentration of M13 in the micelle-poor domains would be orders-of-magnitude greater than that in the micelle-rich domains. Therefore, the effect of entrained micelle-poor, M13-rich domains on the measured concentration of M13 in the macroscopic, micelle-rich, M13-poor phase would be drastic, while the effect of entrained micelle-rich, M13-poor domains on the measured concentration of M13 in the macroscopic, micelle-poor, M13-rich phase would be negligible. Defining $x$ as the volume fraction of micelle-poor domains
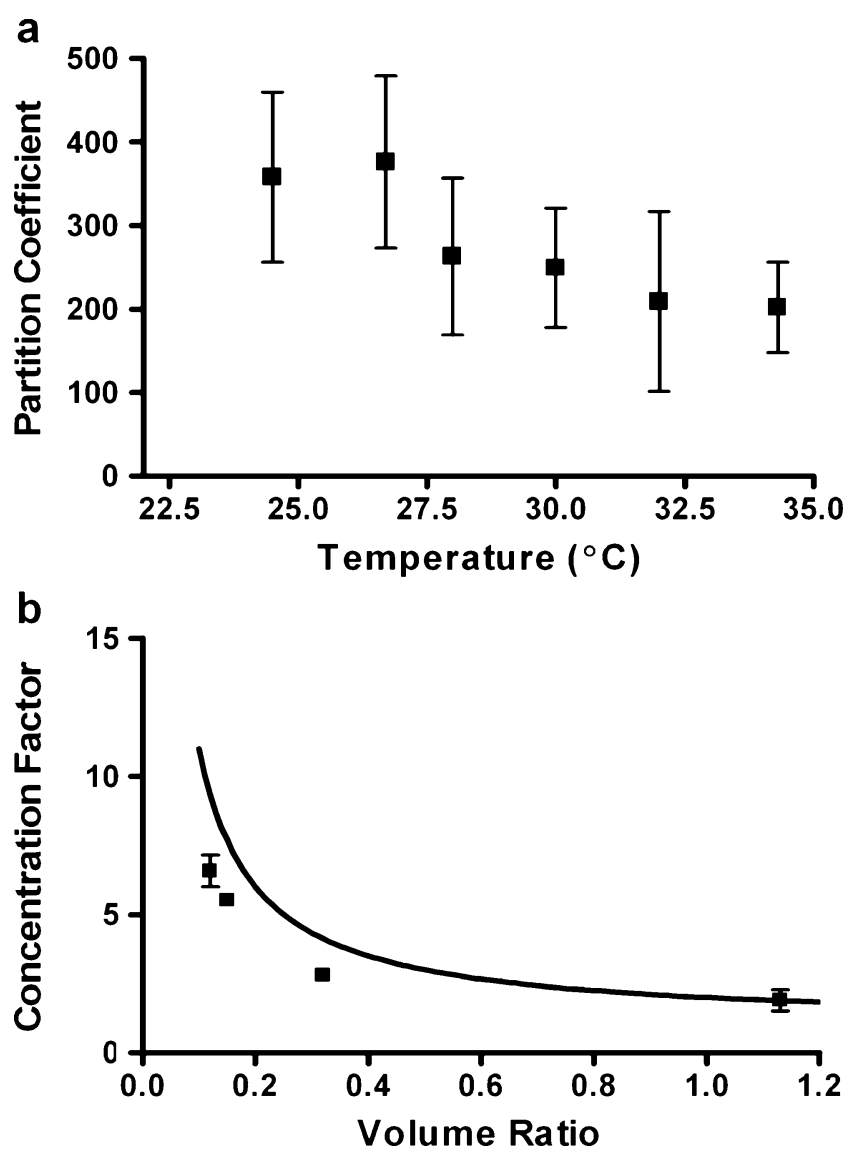

Fig. 2 a Experimentally measured partition coefficients for M13 at various temperatures, and $\mathbf{b}$ comparison of experimentally measured (filled square) and theoretically predicted (solid line) M13 concentration factors for various volume ratios. Error bars represent standard deviations from at least three measurements 


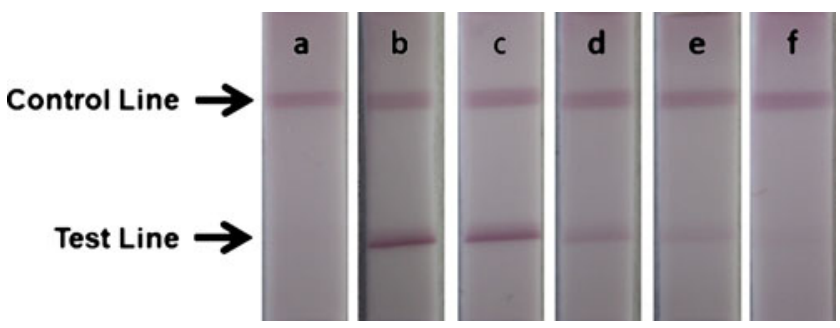

Fig. 3 LFA used to detect M13 without a prior concentration step. The negative control without any M13 is shown in panel a. The remaining solutions contained M13 at concentrations of b $1 \times 10^{10}$, c $5 \times 10^{9}$, d $1 \times 10^{9}$, e $5 \times 10^{8}$, and f $1 \times 10^{8} \mathrm{pfu} / \mathrm{mL}$

entrained in the bottom, macroscopic, micelle-rich phase, the newly predicted partition coefficient could be written as follows $[16,34]$ :

$K_{\mathrm{M} 13}^{\mathrm{EV}+\mathrm{Ent}}=\frac{K_{\mathrm{M} 13}^{\mathrm{EV}}}{1+x \cdot\left(K_{\mathrm{M} 13}^{\mathrm{EV}}-1\right)}$

where $K_{\mathrm{M} 13}^{\mathrm{EV}}$ is the partition coefficient of M13 based only on excluded-volume interactions. For large values of $K_{\mathrm{M} 13}^{\mathrm{EV}}$, as in the case of M13 in ATPMS, Eq. 3 simplifies to:

$\lim _{K_{\mathrm{M} 13}^{\mathrm{EV}} \rightarrow \infty} K_{\mathrm{M} 13}^{\mathrm{EV}+\mathrm{Ent}} \approx \frac{1}{x}$

Equation 4 indicates that the partition coefficient of M13 should only be dependent on $x$. It has also been shown by Blankschtein and coworkers that $x$ is only a function of the volume ratio [34]. Therefore, if the volume ratio is maintained at 1 for all temperatures, the measured partition coefficients for M13 should not change by varying the operating temperature. Figure $2 \mathrm{a}$ shows the partition coefficients of M13 obtained experimentally at various operating temperatures, while the volume ratio was maintained at approximately 1 . As expected and shown in Fig. 2a, the partitioning behavior of M13 in the aqueous two-phase Triton X-114 micellar system is fairly insensitive to the operating temperature, suggesting that M13 partitioning is driven by the excluded-volume interactions between M13 and micelles, but is limited by entrainment. Nevertheless, since the measured values of the M13 partition coefficients are much greater than 1, they can still be exploited as described below.
Concentrating M13 by manipulating the volume ratio

In an approach similar to that developed previously [10], the concentration factor, that is, the concentration of the virus in the top phase divided by the initial concentration, can be expressed by using the following equation [16]:

concentration factor $\equiv \frac{C_{\mathrm{M} 13, \mathrm{t}}}{C_{\mathrm{M} 13,0}}=\frac{\frac{V_{\mathrm{t}}}{V_{\mathrm{b}}}+1}{\frac{V_{\mathrm{t}}}{V_{\mathrm{b}}}+\frac{1}{K_{\mathrm{M} 13}^{m}}}$

where $V_{\mathrm{t}}$ and $V_{\mathrm{b}}$ are the volumes of the top and bottom phases, respectively, $C_{\mathrm{M} 13,0}$ is the initial concentration of M13 in the homogeneous micellar solution prior to phase separation, $C_{\mathrm{M} 13, \mathrm{t}}$ is the concentration of M13 in the top phase, and $K_{\mathrm{M} 13}^{\mathrm{m}}$ is the measured partition coefficient. For large values of $K_{\mathrm{M} 13}^{\mathrm{m}}$, the concentration factor could be approximated as follows:

concentration factor $\approx 1+\frac{1}{\frac{V_{\mathrm{t}}}{V_{\mathrm{b}}}}$

Based on Eq. 6, and the large values of $K_{\mathrm{M} 13}^{\mathrm{m}}$ obtained experimentally (Fig. 2a), the concentration factor can be manipulated by simply varying the volume ratio. Therefore, the volume ratio was varied from 1 to approximately 1/ 8 (0.121), and a two- to sevenfold concentration of M13 in the top phase was achieved (Fig. 2b). In addition, as indicated in Fig. 2b, there is reasonable agreement between the experimentally measured concentration factors and the predictions obtained from Eq. (6).

\section{Detecting M13 via LFA}

After demonstrating that M13 could be concentrated via an ATPMS, we prepared colloidal gold probes and LFA test strips by utilizing goat polyclonal anti-mouse IgG antibody and mouse monoclonal antibody to M13's coat protein pVIII. Figure 3 shows the LFA results. As mentioned previously, the presence of the top line, which contains immobilized goat polyclonal anti-mouse $\operatorname{IgG}$ antibody, indicates a valid test. The presence of the lower test line, which contains mouse monoclonal antibody to M13's coat protein $\mathrm{pVIII}$, indicates the presence of M13. As indicated in Fig. 3, while no test line appeared for the negative
Fig. 4 LFA used to detect M13 with the prior concentration step. The negative control without any M13 is shown in panel a. The remaining solutions initially contained M13 at concentrations of b $1 \times 10^{10}, \mathbf{c} 5 \times$ $10^{9}, \mathbf{d ~} 1 \times 10^{9}$, e $5 \times 10^{8}, \mathbf{f} 1 \times 10^{8}$, g $5 \times 10^{7}$, and h $1 \times 10^{7} \mathrm{pfu} / \mathrm{mL}$

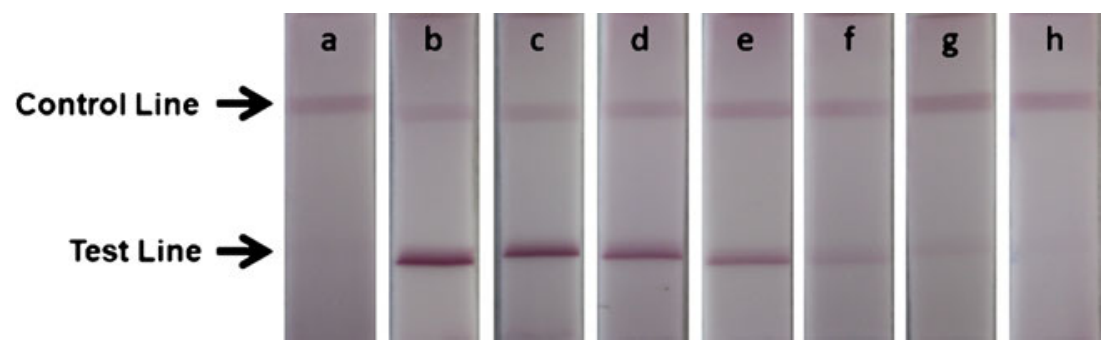


control solution, which did not contain any M13, the intensity of the test line decreased with the decreasing concentration of M13 until the test line was no longer present for the solution containing $10^{8} \mathrm{pfu} / \mathrm{mL}$ (Fig. 3f). This indicated a detection limit of approximately $5 \times 10^{8} \mathrm{pfu} / \mathrm{mL}$ for the M13 LFA performed without a prior concentration step.

\section{Concentrating M13 prior to LFA}

After establishing the detection limit, we investigated the possible improvement of the detection limit if M13 were to be concentrated by utilizing an ATPMS prior to the detection step. To do so, $9.50 \% w / w$ Triton X-114 in PBS solutions with different initial concentrations of M13 were incubated at $26.0{ }^{\circ} \mathrm{C}$ for $18 \mathrm{~h}$. At these operating conditions, a volume ratio of $1 / 9$ was obtained, and based on Eq. 6, an approximately tenfold concentration of M13 in the top, micelle-poor phase was predicted. After phase separation, the top, micelle-poor, M13-rich phases were withdrawn using syringe and needle sets, and were consequently used in the LFA. The results of the LFA with the prior concentration step are shown in Fig. 4. While no test line appeared for the negative control solution, which did not contain any M13, the intensity of the test line decreased with the decreasing concentration of M13 until the test line was no longer present for the solution containing $10^{7} \mathrm{pfu} / \mathrm{mL}$ (Fig. 4h). This indicated a detection limit of approximately $5 \times 10^{7} \mathrm{pfu} / \mathrm{mL}$ for the M13 LFA when combined with the prior concentration step, which represented a tenfold improvement of the detection limit of the LFA assay. Furthermore, the intensity of the test line for all of the detectable concentrations clearly increased when the concentration step was incorporated prior to the detection step. It should be noted that, in this proof-ofconcept study, a volume ratio of only $1 / 9$ was utilized to demonstrate the improvement of the concentration step on LFA's detection limit. In the future, even lower volume ratios may be implemented to yield greater concentration factors that can lead to even lower detection limits. Furthermore, while phase separation of an ATPS could be sped up via low-speed centrifugation [35], since the ultimate goal of this approach is to enhance the detection of pathogens at the point-of-care without using any laboratory equipment, other ATPS could instead be utilized and optimized to achieve rapid separation within the time frame of typical LFA diagnostic tests without the need for centrifugation.

\section{Conclusions}

Concentrating infectious agents, such as infectious viruses, prior to LFA could significantly increase the effectiveness of using LFA for patient management at the POC. This study demonstrated this approach by the novel combination of an ATPMS with LFA to improve the detection limit of bacteriophage M13. The micellar system was generated using the nonionic surfactant Triton X-114 and PBS. We first compared experimentally measured partition coefficients with our theoretical predictions obtained from a model developed previously for cylindrical biomolecules. The agreement between theory and experiment indicated that the partitioning behavior of M13 in the nonionic micellar system is primarily driven by repulsive, steric, excluded-volume interactions that operate between the micelles and M13 particles, but is limited by the entrainment of micelle-poor, M13-rich domains in the macroscopic, micelle-rich phase. Next, the volume ratio was manipulated to concentrate M13 particles in the top phase. By decreasing the volume ratio from 1 to $1 / 8$, M13 particles were concentrated in the top phase two- to sevenfold in a predictive manner. After demonstrating that we could concentrate M13 in the ATPMS, we developed an LFA for the detection of M13 in-solution. The detection limit of the M13 LFA itself was found to be $5 \times 10^{8} \mathrm{pfu} / \mathrm{mL}$. M13 was subsequently concentrated approximately tenfold by utilizing the ATPMS, which led to a tenfold improvement in the LFA detection limit to $5 \times 10^{7} \mathrm{pfu} / \mathrm{mL}$. Therefore, we demonstrated proof-ofprinciple that concentrating a target virus with ATPS prior to the detection step enhances the detection limit of the LFA, thereby increasing the sensitivity of the immunoassay. In the future, the operating conditions could be manipulated to obtain even lower volume ratios, which in turn should result in obtaining higher concentration factors that yield even lower detection limits. Furthermore, the ATPS utilized could be optimized to achieve rapid phase separation within the time frame of typical LFA diagnostic tests, so that the complete detection assay, including the concentration step, could be completed within an hour. We believe once optimized, the novel approach of utilizing ATPS to concentrate target molecules, such as infectious viruses, prior to the detection step could significantly improve the sensitivity of LFA, which in turn could enhance its effectiveness as a POC solution for preventing pandemic outbreaks.

Acknowledgment This work was supported by UCLA funds to D.T.K.

Open Access This article is distributed under the terms of the Creative Commons Attribution Noncommercial License which permits any noncommercial use, distribution, and reproduction in any medium, provided the original author(s) and source are credited.

\section{References}

1. Outbreak of swine-origin influenza A (H1N1) virus infectionMexico, March-April 2009 (2009) J Am Med Assoc 301:23212322 
2. WHO recommendations on the use of rapid testing for influenza diagnosis (2005) World Health Organization. http://www.who.int/ csr/disease/avian_influenza/guidelines/RapidTestInfluenza_web. pdf. Accessed July 2005

3. Uyeki TM, Prasad R, Vukotich C, Stebbins S, Rinaldo CR, Ferng Y, Morse SS, Larson EL, Aiello AE, Davis B, Monto AS (2009) Low sensitivity of rapid diagnostic test for influenza. Clin Infect Dis 48:e89-e92

4. Posthuma-Trumpie GA, Korf J, van Amerongen A (2009) Lateral flow (immuno) assay: its strengths, weaknesses, opportunities and threats. A literature survey. Anal Bioanal Chem 393:569-582

5. Faix DJ, Sherman SS, Waterman SH (2009) Rapid-test sensitivity for novel swine-origin influenza a $(\mathrm{H} 1 \mathrm{~N} 1)$ virus in humans. $\mathrm{N}$ Eng J Med 361:728

6. Cazacu AC, Demmler GJ, Neuman MA, Forbes BA, Chung S, Greer J, Alvarez AE, Williams R, Bartholoma NY (2004) Comparison of a new lateral-flow chromatographic membrane immunoassay to viral culture for rapid detection and differentiation of influenza $\mathrm{a}$ and $\mathrm{b}$ viruses in respiratory specimens. J Clin Microbiol 42:3661

7. Cazacu AC, Greer J, Taherivand M, Demmler GJ (2003) Comparison of lateral-flow immunoassay and enzyme immunoassay with viral culture for rapid detection of influenza virus in nasal wash specimens from children. J Clin Microbiol 41:2132

8. Gavin PJ, Thomson RB (2004) Review of rapid diagnostic tests for influenza. Clin Appl Immunol Rev 4:151-172

9. Ginocchio CC, Zhang F, Manji R, Arora S, Bornfreund M, Falk L, Lotlikar M, Kowerska M, Becker G, Korologos D (2009) Evaluation of multiple test methods for the detection of the novel 2009 influenza a (H1N1) during the New York City outbreak. J Clin Virol 45:191-195

10. Albertsson PA (1986) Partition of cell particles and macromolecules, 3rd edn. Wiley, New York

11. Quina FH, Hinze WL (1999) Surfactant-mediated cloud point extractions: an environmentally benign alternative separation approach. Ind Eng Chem Res 38:4150-4168

12. Albertsson PA (1989) Separation of cells and cell organelles by partition in aqueous polymer two-phase systems. Methods Enzymolog 171:532-549

13. Kamei DT, King JA, Wang DI, Blankschtein D (2002) Separating lysozyme from bacteriophage p22 in two-phase aqueous micellar systems. Biotechnol Bioeng 80:233-236

14. Liu CL, Kamei DT, King JA, Wang DI, Blankschtein D (1998) Separation of proteins and viruses using two-phase aqueous micellar systems. J Chromatogr B 711:127-138

15. Ribeiro SC, Monteiro GA, Cabral JM, Prazeres DM (2002) Isolation of plasmid DNA from cell lysates by aqueous two-phase systems. Biotechnol Bioeng 78:376-384

16. Mashayekhi F, Meyer AS, Shiigi SA, Nguyen V, Kamei DT (2009) Concentration of mammalian genomic DNA using twophase aqueous micellar systems. Biotechnol Bioeng 102:16131623
17. Champluvier B, Kula MR (1992) Sequential membrane-based purification of proteins, applying the concept of multidimensional liquid chromatography (MDLC). Biosep 2:343-351

18. Lindblom H (1983) Rapid chromatographic method for the isolation of glucose-6-phosphate dehydrogenase from yeast enzyme concentrate. J Chromatogr A 266:265-271

19. Lindblom H, Soderberg L, Cooper EH, Turner R (1983) Urinary protein isolation by high-performance ion-exchange chromatography. J Chromatogr A 266:187-196

20. Tanford C (1978) The hydrophobic effect: formation of micelles and biological membranes, p1-10, 2nd edn. Wiley, New York

21. Bordier C (1981) Phase separation of integral membrane proteins in triton x-114 solution. J Biol Chem 256:1604-1607

22. Nikas YJ, Liu C-L, Srivastava T, Abbott NL, Blankschtein D (1992) Protein partitioning in two-phase aqueous nonionic micellar solutions. Macromol 25:4797-4806

23. Carlson K (2005) Appendix: Working with bacteriophages: Common techniques and methodological approaches. In: Kutter E, Sulakvelidze A (eds) Bacteriophages: biology and applications. CRC, Boca Raton

24. Frens G (1972) Particle size and sol stability in metal colloids. Colloid Polym Sci 250:736-741

25. Horisberger M, Clerc MF (1985) Labelling of colloidal gold with protein A. A quantitative study. Histochem 82:219-223

26. Leuvering JH, Thal PJ, van der Waart M, Schuurs AH (1980) Sol particle immunoassay (SPIA). J Immunoass 1:77-91

27. Lazzara MJ, Blankschtein D, Deen WM (2000) Effects of multisolute steric interactions on membrane partition coefficients. J Colloid Interface Sci 226:112-122

28. Day LA, Marzee CJ, Reisberg SA, Casadevall A (1988) DNA packing in filamentous bacteriophages. Annu Rev Biophys Biophys Chem 17:509-539

29. Won YY, Davis HT, Bates FS (1999) Giant wormlike rubber micelles. Sci 283:960-963

30. Robson RJ, Dennis EA (1977) The size, shape, and hydration of nonionic surfactant micelles. Triton X-100. J Phys Chem 81:1075-1078

31. Acharya DP, Kunieda H (2003) Formation of viscoelastic wormlike micellar solutions in mixed nonionic surfactant systems. J Phys Chem B 107:10168-10175

32. Paradies HH (1980) Shape and size of a nonionic surfactant micelle. Triton X-100 in aqueous solution. J Phys Chem 84:599-607

33. Dalhaimer P, Bates FS, Discher DE (2003) Single molecule visualization of stable, stiffness-tunable, flow-conforming worm micelles. Macromol 36:6873-6877

34. Kamei DT, King JA, Wang DI, Blankschtein D (2002) Understanding viral partitioning in two-phase aqueous nonionic micellar systems: 2. Effect of entrained micelle-poor domains. Biotechnol Bioeng 78:203-216

35. Albertsson PA (1970) Partition of cell particles and macromolecules in polymer two-phase systems. Adv Protein Chem 24:309-341 